\title{
Field Demonstration of a Tunable WDM-PON System with Novel SFP+ Modules and Centralized Wavelength Control
}

\author{
S. Pachnicke ${ }^{1}$, S. Mayne ${ }^{2}$, B. Quemeneur ${ }^{2}$, D. Sayles ${ }^{2}$, H. Schwuchow ${ }^{3}$, J. Zhu ${ }^{4}$, A. Wonfor ${ }^{4}$, \\ P. Marx ${ }^{5}$, M. Lawin ${ }^{1}$, M. Fellhofer ${ }^{5}$, R. Turner ${ }^{2}$, P. Neuber ${ }^{3}$, M. Dietrich ${ }^{3}$, M. J. Wale ${ }^{2}$, \\ R. V. Penty ${ }^{4}$, I. H. White ${ }^{4}$, and J.-P. Elbers ${ }^{1}$ \\ ${ }^{1}$ ADVA Optical Networking SE, Maerzenquelle 1-3, 98617 Meiningen, Germany \\ ${ }^{2}$ Oclaro Technology Ltd., Long Road, Paignton, Devon, TQ4 7AU, United Kingdom \\ ${ }^{3}$ ELCON Systemtechnik GmbH, Obere Hauptstr. 10, 09232 Hartmannsdorf, Germany \\ ${ }^{4}$ Centre for Photonic Systems, University of Cambridge, 9 JJ Thomson Avenue, Cambridge, CB3 OFA, United Kingdom \\ ${ }^{5}$ Energie AG Oberösterreich Data GmbH, Böhmerwaldstr. 3, 4021 Linz, Austria \\ spachnicke@advaoptical.com
}

\begin{abstract}
We report on a demonstration of a novel tunable WDM-PON system over $25 \mathrm{~km}$ of field deployed fiber. We show error-free operation at $1 \mathrm{GbE}$ with sensitivity better than $-30 \mathrm{dBm}$ and centralized control of the ONU wavelengths.

OCIS codes: (060.2330) Fiber optics communications; (140.3600) Lasers, tunable
\end{abstract}

\section{Introduction}

Several passive optical network (PON) architectures and technologies have been investigated over the past years for providing broadband optical access. Next generation systems will likely also be utilized for mobile back- and fronthauling as well as data center connectivity. The combined requirements for multi-service support, high bit rates and increased reach lead to the use of DWDM in next-generation access systems [1]. This is also reflected by the outcome of the FSAN NG-PON2 discussion and related ITU-T recommendation G.989.2 [2]. As a special form wavelength routed WDM-PON systems make use of a wavelength-selective filter in the remote node (typically an arrayed-waveguide grating, AWG). A cyclic AWG at the remote node allows single-fiber operation of the WDMPON system with downstream (DS) channels in the L-band and upstream (US) channels in the C-band. For operational simplicity and cost wavelength agnostic, colorless devices are required in the optical network units (ONUs).

In this paper, we demonstrate a WDM-PON system with novel SFP+ form-factor tunable transceiver modules in the ONU. These are based on a novel high temperature Aluminium containing (AlInGaAs) DBR laser [3] and can operate without individual wavelength lockers. The modules are integrated into newly developed customer premises equipment (CPE) supporting symmetric $1 \mathrm{GbE}$ operation. The ONU wavelengths are managed by a central wavelength controller in the optical line terminal (OLT) to enable automatic $\lambda$-tuning on power-up and long-term

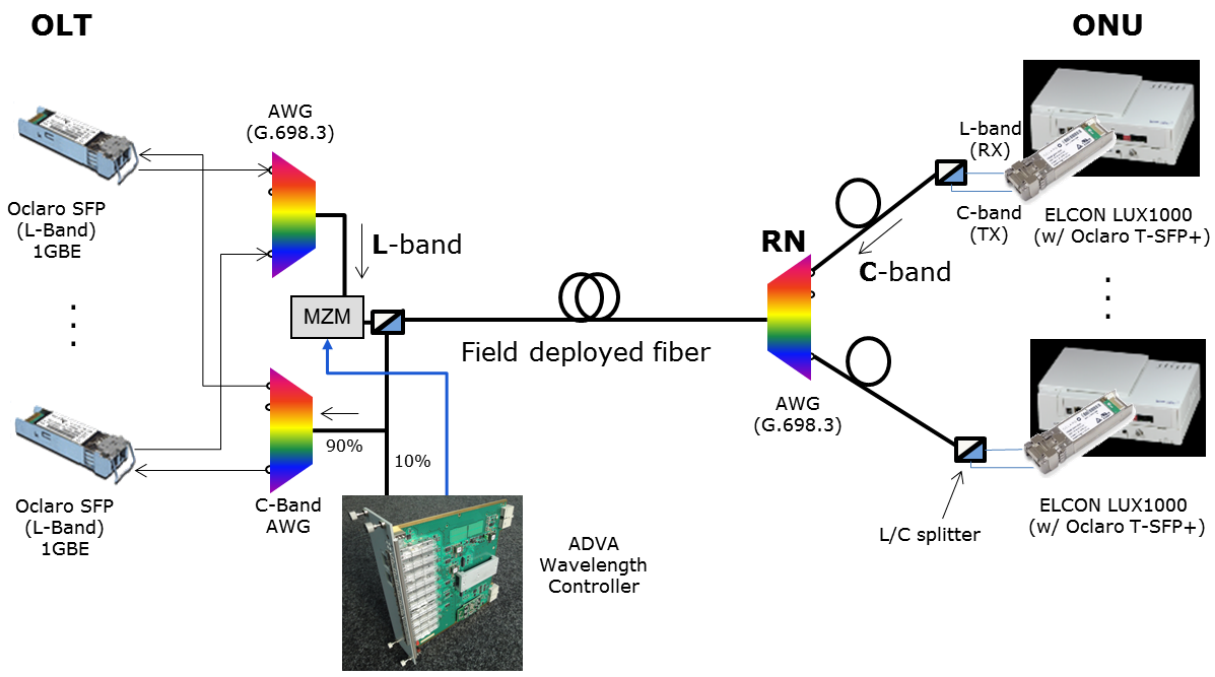

Fig. 1. Experimental setup of tunable WDM-PON system with centralized wavelength control (OLT: optical line termination, RN: remote node, ONU: optical network unit, MZM: Mach-Zehnder modulator) 
wavelength stability. Feedback is carried by a tone-based embedded communication channel. To the best of our knowledge, this is the first paper that reports on such a prototypical tunable WDM-PON system in a field-trial scenario with all functions realized in embedded hardware.

\section{Field Trial Setup}

A schematic of the WDM-PON system under investigation is depicted in Fig. 1. In the OLT a field programmable gate array (FPGA) connected to a wavelength locker is used to compute feedback information on wavelength accuracy and power levels of all attached ONUs ("centralized wavelength controller"). This is facilitated by simultaneously detecting distinct c.w. pilot-tones (here lying in the range between $100 \mathrm{kHz}$ and $190 \mathrm{kHz}$ ) from each ONU laser at a shared etalon in the OLT.

Feedback information is modulated onto a $100 \mathrm{kHz}$ pilot tone with $1 \mathrm{~kb} / \mathrm{s}$ ASK modulation and transported back to the ONUs. In the field trial the pilot-tone was applied to the (DS) WDM signal by use of a Mach-Zehnder modulator (approx. 10\% modulation depth). In a later product a variable optical attenuator (VOA) might be used instead to eliminate polarization sensitivity. At the ONU the tone is detected by the received signal strength indicator (RSSI) of the SFP+ and decoded by an external microcontroller embedded in the CPE on a separate subboard. The tuning algorithm [4] is also implemented on this microcontroller to control the different sections of the (US) DS-DBR laser. The (US) c.w. pilot-tone is applied by modulation of the SFP's SOA current (yielding approx. $10 \%$ modulation depth) and is also generated by the microcontroller driving a direct digital synthesis (DDS) chip. In the experiment three neighboring channels have been used for DS and US (on a $100 \mathrm{GHz}$ grid). These were modulated by an Ethernet tester with $1 \mathrm{GbE}$ data streams (PRBS $2^{31}-1$ in the payload). The SFP+ modules would allow in theory operation up to $10 \mathrm{~Gb} / \mathrm{s}$; however, this has not been tested here due to availability of only a $1 \mathrm{GbE}$ CPE (lab measurements on the physical layer performance of the SFP+ at $10 \mathrm{GbE}$ line rate are reported in [5]). The output power of the tunable ONU lasers was approximately $0 \mathrm{dBm}$. The employed (cyclic) AWGs meet the ITU-T recommendation G.698.3 with $100 \mathrm{GHz}$ channel spacing in C-band and $97.15 \mathrm{GHz}$ spacing in L-band. In DS direction standard (fixed-wavelength) L-band SFPs (approx. $5.5 \mathrm{dBm}$ output power) have been used, which have been slightly detuned to meet the $97.15 \mathrm{GHz}$ grid. The optical distribution network (ODN) had a reach of $15 \mathrm{~km}$ (7 dB fiber loss) or $25 \mathrm{~km}$ (11 dB fiber loss) as shown in Fig. 2. The rather high attenuation is caused from a high number of patches.

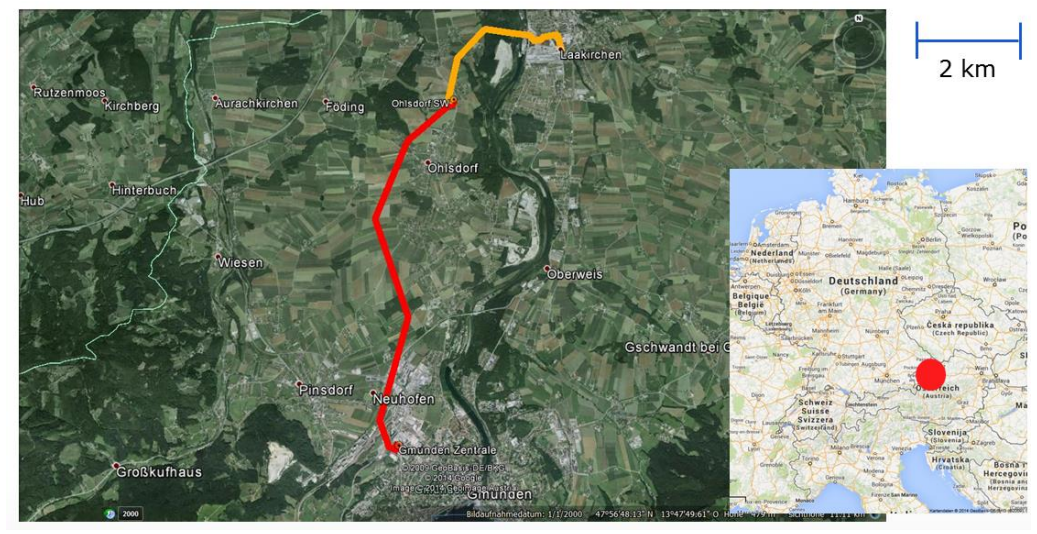

Fig. 2. Map showing the two different fiber routes of $15 \mathrm{~km}$ (red) and $25 \mathrm{~km}$ (red+orange) lengths in Energie AG Oberösterreich Data network

\section{Wavelength-Tuning Algorithm}

The laser control algorithm $[3,4]$ is used in two stages. It allows plug \& play configuration of the ONUs by tuning them automatically to the right frequency (according to the AWG port they are attached to). On power-up of the ONU a frequency sweep is initiated to find the appropriate AWG wavelength slot. Crosstalk to neighboring channels is suppressed by the AWG transfer function and no increase in BER of the neighboring channels could be observed during this frequency sweep. In this phase, only the received power level is used as a feed-back criterion until a pre-defined threshold of the received signal power is exceeded. This indicates that the current laser frequency is lying in the desired AWG pass-band slot. As soon as the power threshold is reached the sweep step width is reduced. In this second phase, the control information from the centralized etalon is used to fine tune the laser wavelength and to keep it at its desired frequency with a high accuracy and ensure single mode operation. 


\section{Results}

In all our measurements the ONU laser operated at a temperature of approx. $58^{\circ} \mathrm{C}$. In a previous publication [3] we have shown that also free-running operation in an even higher temperature range of $70^{\circ} \mathrm{C}$ to $90^{\circ} \mathrm{C}$ is possible (which can be maintained by a simple heater).

In the field trial we first investigated the ONU frequency stability (after it has automatically tuned to its desired frequency slot). The accuracy was better than $\pm 5 \mathrm{GHz}$ (Fig. 3, left) and maintained for more than 9 hours. In a second measurement the sensitivity at the receiver has been evaluated (Fig. 3, right) for the two different reaches of $15 \mathrm{~km}$ and $25 \mathrm{~km}$. There is no obvious difference in sensitivity for these two transmission distances. In the upstream direction the sensitivity was approx. $-34 \mathrm{dBm}$ for error-free operation. In the downstream direction the sensitivity was approximately $4 \mathrm{~dB}$ less $(-30 \mathrm{dBm})$. The reason for the difference is that at the OLT side the signal is received by a standard SFP, which is optimized for $1 \mathrm{GbE}$ operation, whereas in DS direction the signal is received by the $\mathrm{SFP}+$, which is designed for operation of up to $10 \mathrm{GbE}$. A penalty about $1 \mathrm{~dB}$ has been observed due to insertion of the pilot-tone.
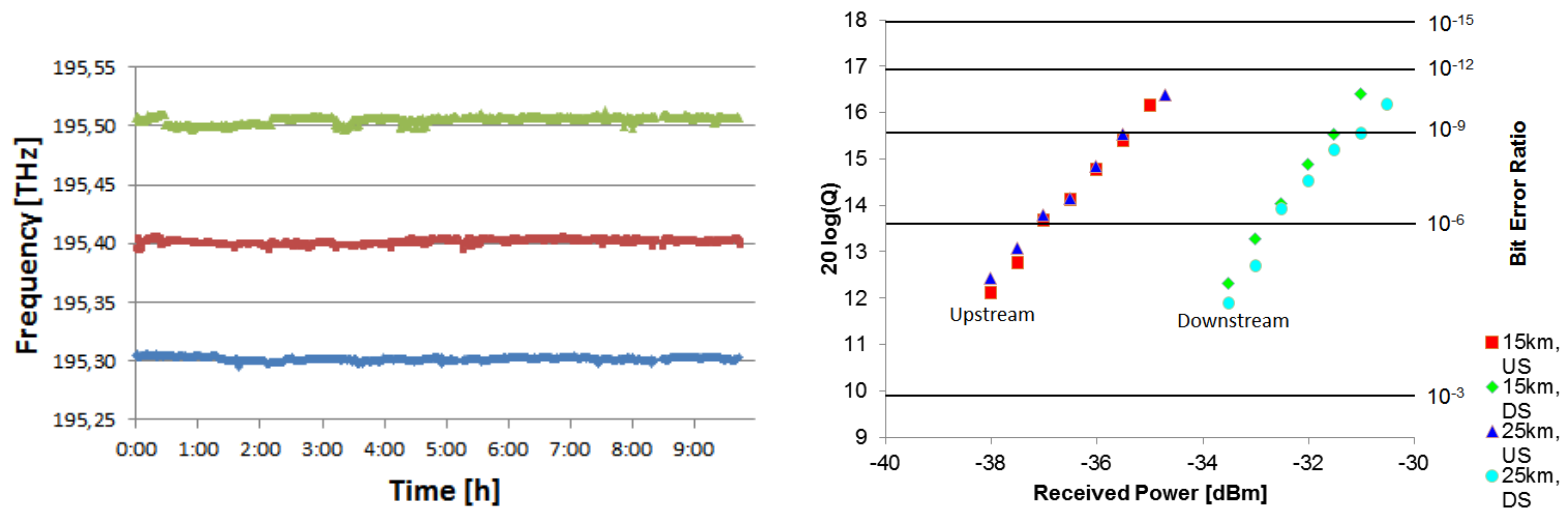

Fig. 3. Long-term frequency stability of three upstream channels (left) and measured BER for $1 \mathrm{GbE}$ up-/ and downstreams and different ODN reaches (right)

\section{Summary and Conclusions}

We have demonstrated for the first time a tunable WDM-PON system in a field trial scenario with a novel SFP+ based ONU transceiver, which was plugged into a prototypical $1 \mathrm{GbE} \mathrm{CPE}$ and used centralized wavelength locking with all functionality embedded in hardware (FPGA and microcontroller). The field test has demonstrated that automatic tuning of the ONU to the desired frequency slot (i.e. AWG port) is feasible, and long-term frequency stability can be achieved by centralized wavelength locking. Communication between OLT and ONU was realized with a pilot-tone based embedded communication channel, which is protocol-independent. Error-free transmission of $1 \mathrm{GbE}$ data was demonstrated successfully at reaches up to $25 \mathrm{~km}$ with sensitivity in excess of $30 \mathrm{dBm}$. Thus the system supports an ODN budget of approx. $18 \mathrm{~dB}$ in the downstream and approx. $20 \mathrm{~dB}$ in the upstream direction, respectively.

\section{Acknowledgements}

This work has been supported by the EU ERA-NET+ projects PIANO+ IMPACT and PIANO+ TUCAN co-funded by the German BMBF, the UK Technology Strategy Board and the Austrian FFG. Also support from the UK EPSRC INTERNET project is acknowledged.

\section{References}

[1] K. Grobe, et al, “Access Networks based on Tunable Lasers”, IEEE J. Lightw. Technol. 32, 2815-2823 (2014).

[2] 40-Gigabit-capable passive optical networks (NG-PON2): PMD layer specifications, ITU-T G.989.2 (2013).

[3] S. Pachnicke, et al, "First Demonstration of a Full C-Band Tunable WDM-PON System with Novel High-Temperature DS-DBR Lasers", Proc. of OFC, W3G.2 (2014).

[4] J. Zhu, et al, "Athermal Colourless C-band Optical Transmitter for Passive Optical Networks", accepted for publication in IEEE J. Lightw. Technol. (2014).

[5] J. Zhu, et al, "First demonstration of a WDM-PON system using novel full C-band tunable SFP+ transceiver modules [Invited]", IEEE/OSA J. Opt. Comm. Netw. 7, A28-A36 (2015). 